\title{
Influence of antenatal steroids and sex on maturation of the epidermal barrier in the preterm infant
}

Anoo Jain, Nicholas Rutter, Patrick H T Cartlidge

\begin{abstract}
Background-The epidermal barrier is well developed in term infants but defective in the immature infant with important clinical consequences. The development of the barrier shares similarities with production of pulmonary surfactant. Studies in the rat have shown that barrier maturation is accelerated by antenatal steroids, both structurally and functionally. Females have a more mature barrier than males at the same gestational age. These factors have not been studied in the human.
\end{abstract}

Aim-To examine the influence of antenatal steroids and sex on maturation of the epidermal barrier in the preterm infant.

Subjects-A total of 137 infants born before 34 weeks gestation, 80 boys and 57 girls, were studied: 87 had been exposed to antenatal steroids, and 50 had not; 99 were studied prospectively, and 38 had been studied previously.

Method-Barrier function was measured as transepidermal water loss from abdominal skin by evaporimetry. Measurements were made within the first 48 hours and corrected to a standard relative humidity of $50 \%\left(\mathrm{TEWL}_{50}\right)$.

Results-The relation between TEWL $_{50}$ and gestation was exponential with very high levels in the most immature infants. No influence of antenatal steroids or sex could be shown. When infants who were optimally exposed to antenatal steroids were considered alone, no effect could be shown.

Academic Division of Child Health, Nottingham City Hospital, University of Nottingham, Hucknall Road, Nottingham

NG5 1PB, UK

A Jain

N Rutter

Department of Child Health, University of Wales College of Medicine, Cardiff CF14 4XW, UK

P H T Cartlidge

Correspondence to: Professor Rutter, Academic Division of Child Health, School of Human

Development, Queen's Medical Centre, Nottingham NG7 2UH, UK

email:

Nick.Rutter@nottingham.ac.uk

Accepted 4 May 2000 mis, the stratum corneum. Lipid bilayers fill the intercellular spaces between the corneocyte cell plates like the mortar between bricks. ${ }^{6}$ The lipid is produced by lamellar bodies in the cytoplasm of the cells of the stratum granulosum, the cell layer immediately below the stratum corneum. The contents of the lamellar bodies are extruded into the intercellular spaces. The whole process has similarities to production of pulmonary surfactant and may therefore be expected to be influenced by the antenatal administration of steroids. In the animal model, antenatal steroid administration has a considerable effect on epidermal barrier development, both structural and functional. ${ }^{78}$ There are therefore reasons to suppose that in the human there may be a similar maturational effect, but this has never been examined.

Animal studies have also shown an effect of sex on epidermal barrier maturation as well as pulmonary maturation. ${ }^{9}$ Females have a more mature barrier than males at the same gestational age. The influence of sex on epidermal maturation in the human infant has not been examined.

We carried out this study to see if antenatal steroids have a maturational effect on the human newborn epidermis. Because the use of antenatal steroids is now so widespread and effective, a randomised controlled trial was clearly impossible. The study was therefore a prospective observational one, comparing epidermal barrier function in preterm infants exposed to steroids with contemporary and historical controls who were not. We also examined the influence of sex.

\section{Subjects}

The policy in our maternity unit is to give steroids in the form of dexamethasone, two doses 12 hours apart (total dose $24 \mathrm{mg}$ ), to all mothers in whom delivery before 34 weeks gestation is predicted. In all cases, the mother's treatment card was directly inspected to see whether steroids had been given and to note the timing in relation to the delivery. Gestational age was calculated from the expected date of delivery predicted by early ultrasound examination. If this date differed from the expected date of delivery calculated from the last menstrual period by more than two weeks, the infant was excluded from the study.

Ninety nine unselected infants born before 34 weeks gestation were studied in the newborn intensive care units of Nottingham City Hospital and Queens Medical Centre, 
Table 1 Details of the infants and ambient measurement conditions

\begin{tabular}{llllll}
\hline & $\begin{array}{l}\text { Number of } \\
\text { infants }(M / F)\end{array}$ & $\begin{array}{c}\text { Gestation } \\
\text { (weeks) }\end{array}$ & Birth weight $(k g)^{*}$ & $\begin{array}{c}\text { Ambient relative } \\
\text { humidity (\%) } t\end{array}$ & $\begin{array}{c}\text { Ambient temperature } \\
\left({ }^{\circ} \mathrm{C}\right) \dagger\end{array}$ \\
\hline Any steroids & $87(48 / 39)$ & $30(25-33)$ & $1.31(0.56-2.67)$ & $34.7(9-70)$ & $35.3(32.0-37.9)$ \\
No steroids & $50(32 / 18)$ & $29(25-33)$ & $1.33(0.73-1.53)$ & $32.6(16-62)$ & $34.8(27.0-37.3)$ \\
$\quad$ Contemporary & $12(9 / 3)$ & $30(26-33)$ & $1.47(0.93-2.01)$ & $30.2(16-60)$ & $35.2(31.9-37.1)$ \\
Historical & $38(23 / 15)$ & $29(25-33)$ & $1.28(0.73-2.58)$ & $44.3(31-62)$ & $33.4(27.0-37.1)$ \\
\hline
\end{tabular}

*Values are median (range).

tValues are mean (range).

Nottingham between September 1997 and September 1998. We excluded infants with diseased or damaged skin and those transferred to another unit early in the postnatal period. Table 1 gives the details of the infants studied. Because of the appreciable effect of gestation on TEWL, we divided subjects by gestation into those above and below 30 weeks. In total, 87 infants had received at least one dose of steroids before delivery, and 43 of these had received steroid treatment considered optimal in terms of pulmonary maturation - that is, two doses of dexamethasone more than 24 hours but less than one week before delivery. The remaining 44 received suboptimal treatmentone or more doses of dexamethasone within 24 hours or more than a week before birth. Twelve infants in this contemporary group did not receive any antenatal steroids.

Because antenatal steroid use is so high, we included for comparison a group of 38 preterm infants who were studied in our unit in $1985 / 1986$ at a time when antenatal steroids were not used. These infants were studied in the same way. Abdominal TEWL measurements were made as part of a study on percutaneous gas exchange in the newborn and have not previously been published. Gestation ranged from 25 to 33 weeks (median 29) and birth weight from 0.73 to $2.58 \mathrm{~kg}$ (median 1.28). In each case, ambient relative humidity and temperature were recorded. These 38 infants were combined with the 12 in the contemporary study to form a no steroid group of 50 infants. The two groups, steroid and no steroid, were similar in gestation and birth weight (table 1). Of the 137 infants in the study, 80 were male. No infant received steroids in the early postnatal period during the time of study.

\section{Methods}

Epidermal barrier function was measured directly as TEWL using an Evaporimeter (Servomed, Sweden). ${ }^{10}$ This non-invasive technique estimates TEWL by measurement of the gradient of water vapour pressure close to the skin. It has been widely used in the newborn and in animals. The probe containing two water vapour pressure sensors is held lightly against the skin until a stable reading of TEWL $\left(\mathrm{g} / \mathrm{m}^{2} / \mathrm{h}\right)$ is obtained. The probe also records ambient relative humidity. Infants were studied lying supine in an incubator. Ambient relative humidity was not controlled, but was measured in the centre of the incubator above the infant. Added humidity from an external humidifier was routinely used in the nursing care of all infants below $1 \mathrm{~kg}$ in the first week of life. The humidifier was turned off and the incubator allowed to run dry for about an hour before TEWL was measured.

TEWL was measured from abdominal skin. Previous studies have shown that this is an area of particularly high TEWL in the preterm infant. ${ }^{4}$ It is also a convenient site for measurement as it is accessible in sick infants without interference and the skin is flat. TEWL was measured from undamaged skin between the xiphisternum and umbilicus in the midline, by a single observer. The incubator air temperature was recorded. TEWL from the historical controls was measured in the same way by a single observer using the same Evaporimeter. In the contemporary infants, measurements were made on three occasions at about eight, 24 , and 48 hours after delivery. After correction to a standard relative humidity (see below), these were averaged to obtain a mean value for the first 48 hours. In the historical infants, a single measurement was made within the first 48 hours (median age 24 hours, range 4 to 48).

Ambient humidity has an appreciable effect on TEWL. The relation is an inverse linear one, with zero TEWL at $100 \%$ relative humidity. ${ }^{31-13}$ Because measurements were made over a wide range of ambient relative humidity $(9-70 \%)$, we corrected them to a standard relative humidity of $50 \%\left(\mathrm{TEWL}_{50}\right)$ using the following equation:

$\mathrm{TEWL}_{\mathrm{m}}=\left(100-\mathrm{RH}_{\mathrm{a}}\right) / \mathrm{s}$

where TEWL $_{\mathrm{m}}$ is the measured transepidermal water loss, $\mathrm{RH}_{\mathrm{a}}$ is the ambient relative humidity, and $s$ is the slope of the line that relates the two. $\mathrm{s}$ is calculated and hence: TEWL $_{50}=50 / \mathrm{s}$

Values of TEWL $_{50}$ therefore reflect differences in epidermal barrier function and not the environmental conditions. As TEWL $\mathrm{T}_{50}$ is considerably affected by gestation, the values are not normally distributed. After log transformation, the effects of antenatal steroids and sex were examined by stepwise linear regression, with $\log$ TEWL $_{50}$ as the response variable. Gestation groups were compared using nonparametric testing (Mann-Whitney U).

\section{Results}

The relation between TEWL $_{50}$ and gestation is exponential, with very high levels of TEWL in the most immature infants. Figure 1 shows values for TEWL $_{50}$ plotted on a log scale against gestational age. The line of best fit is linear. When the linear regression lines for the infants receiving antenatal steroids and the control infants are compared, they are virtually identical. Stepwise linear regression showed that the only variable that influenced TEWL $_{50}$ 
Steroids

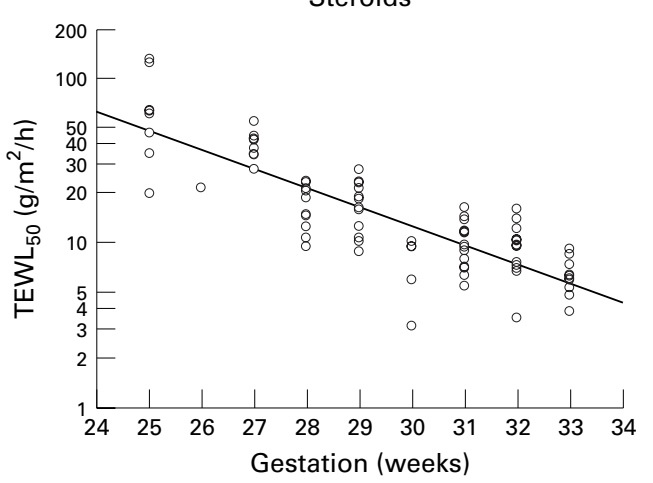

No steroids

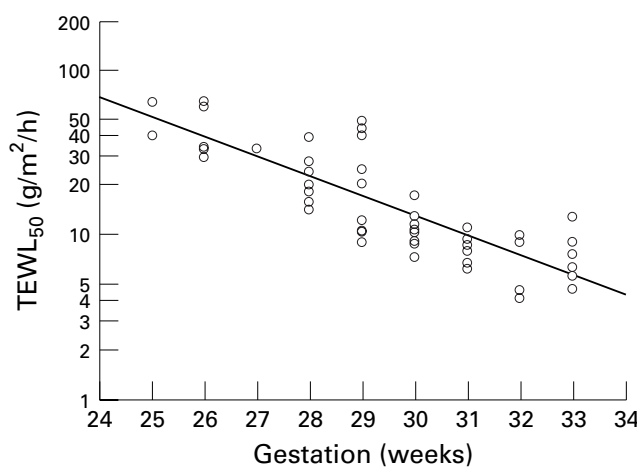

Figure 1 Relation between transepidermal water loss $\left(T E W L_{50}\right)$ and gestational age in infants exposed (upper graph) or not (lower graph) to antenatal steroids. The vertical axis is a log scale, and the regression lines are shown. They are not significantly different.

Table 2 Median values of TEWL $L_{50}$ in the two gestational age groups compared by exposure to antenatal steroids

\begin{tabular}{lccc}
\hline & & $T E W L_{50}\left(g / m^{2} / h\right)$ \\
\cline { 3 - 4 } Gestation & $n$ & Median & Interquartile range \\
\hline 24-29 weeks & & & \\
Any ANS & 40 & 22.5 & $15.1-41.5$ \\
$\quad$ Optimal ANS & 17 & 23.2 & $14.6-44.6$ \\
$\quad$ Suboptimal ANS & 23 & 21.9 & $15.8-42.9$ \\
No ANS & 25 & 30.3 & $17.1-40.7$ \\
30-34 weeks & & & \\
Any ANS & 47 & 8.0 & $6.3-10.4$ \\
$\quad$ Optimal ANS & 26 & 7.5 & $6.2-10.3$ \\
$\quad$ Suboptimal ANS & 21 & 9.2 & $6.4-11.2$ \\
No ANS & 25 & 9.0 & $6.5-10.6$ \\
\hline $\begin{array}{l}\text { Optimal and suboptimal ANS are defined in the text. } \\
\text { TEWL }\end{array}$, transepidermal water loss corrected to a standard \\
relative humidity of 50\%; ANS, antenatal steroids.
\end{tabular}

was gestational age; antenatal steroids had no effect. When steroid and non-steroid exposure were compared in the two gestational age groups, median values were not significantly different (table 2). No significant difference between median values was seen either when the steroid treated infants were subdivided into optimal and suboptimal groups (table 2). No maturational effect of antenatal steroids could therefore be shown.

As no maturational effect of steroids could be shown, we combined the steroid and no steroid groups to examine the influence of sex

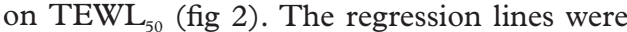
compared in boys and girls and found to be almost identical. Again stepwise linear regression showed that the only variable that influenced $\mathrm{TEWL}_{50}$ was gestational age; sex
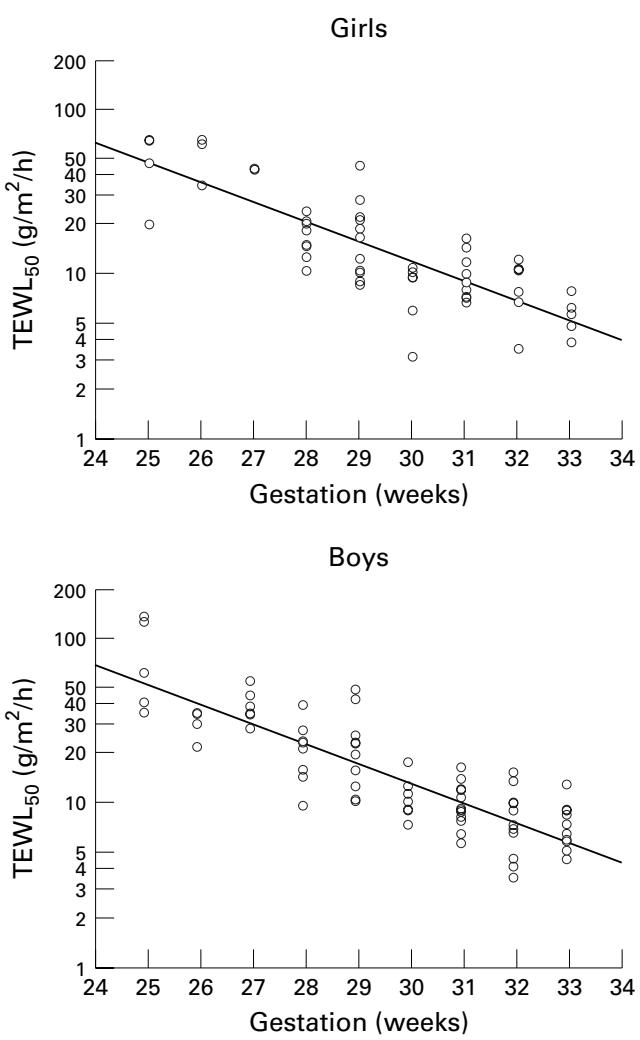

Figure 2 Relation between transepidermal water loss $\left(T E W L_{50}\right)$ and gestational age in girls (upper graph) and boys (lower graph). The vertical axis is a log scale, and the regression lines are shown. They are not significantly different.

Table 3 Median values for transepidermal water loss corrected to a standard relative humidity of $50 \%$

$\left(T E W L_{5}\right)$ in the two gestational age groups compared by sex

\begin{tabular}{llll}
\hline & & \multicolumn{2}{l}{$T E W L_{50}\left(g / m^{2} / h\right)$} \\
\cline { 3 - 4 } Gestation & $n$ & Median & Interquartile range \\
\hline 24-29 weeks & & & \\
$\quad$ Male & 35 & 28.5 & $19.5-39.3$ \\
Female & 30 & 20.5 & $14.0-43.9$ \\
30-34 weeks & & & \\
$\quad$ Male & 45 & 9.0 & $6.5-10.7$ \\
Female & 27 & 7.7 & $6.2-10.4$ \\
\hline
\end{tabular}

had no effect. When boys and girls were compared in the two gestational age groups, median values were not significantly different (table 3).

\section{Discussion}

Antenatal steroids have a maturational effect on organs other than the lung, notably the gut and kidneys. ${ }^{14-18}$ The similarity between alveolar surfactant and intercellular epidermal lipid is striking, so a maturational effect of antenatal steroids on the epidermal barrier could be predicted. ${ }^{19}$ Aszterbaum et al explored this in the rat model. Maturation was assessed in terms of gross structure, ultrastructure, lipid composition, and barrier function (as TEWL). They found that, if the mother was given betamethasone between days 16 and 18 and the pups were delivered on day 19 (term is $21-22$ days ), the epidermal barrier showed maturation to term levels compared with untreated 
controls. Similar results in the rat were obtained by Okah et al. ${ }^{8}$ This is equivalent to the maturational effect of several weeks of gestation in utero or one to two weeks of postnatal existence in the human. The effect of antenatal steroids on human epidermal development has not been examined before.

Our study is limited by its design, because a randomised controlled trial of antenatal steroids would clearly be impossible. However, we think our use of contemporary and historical controls is valid. Antenatal steroid use is now very extensive, and our contemporary preterm infants who were not exposed to steroids are therefore a selected group in whom steroid administration was not possible. The historical controls were unselected and were no different from the 99 infants studied recently in terms of gestation, age at study, method of measurement of TEWL, and environmental conditions. Although the average and range of ambient relative humidity was similar in the steroid and non-steroid groups and in the contemporary and historical groups, the effect of humidity on TEWL is so great that we corrected the results to a standard relative humidity of $50 \%$ $\left(\right.$ TEWL $\left._{50}\right)$ as others have done. ${ }^{20}$ Other environmental influences such as ambient temperature have a very small effect on TEWL. Differences in values of TEWL ${ }_{50}$ between individual infants are therefore a result of differences in the effectiveness of the epidermal barrier. We can be sure that the historical controls were not exposed to antenatal steroids.

Why have we have failed to show any maturational effect of antenatal steroids on the epidermal barrier? It is not likely to be the result of poor timing of their administration in relation to delivery. The optimum timing of steroids for reduction in mortality and respiratory morbidity is between 24 and 168 hours before birth. ${ }^{21}$ When we compared these infants with the untreated group, there were no differences in TEWL $_{50}$. It is not clear why the newborn human epidermis behaves differently from the rodent epidermis because in several other respects they are similar. On the basis of the animal epidermal model and the effect of antenatal steroids on pulmonary surfactant in the human, we were looking for a major maturational effect (equivalent to several weeks of gestation), similar to the postnatal maturation that occurs in immature infants in response to being born. ${ }^{412}$ Such an effect would be seen as a shift of the regression line to the right in fig 1 . The antenatal steroid regimen used in the animal studies was different from that used for pulmonary maturation in the human - the dose of betamethasone was higher and it was given for longer - but this seems unlikely to explain the lack of effect. We conclude that there is a species difference in epidermal maturation.

Hanley et al showed a sex difference in epidermal maturation in the fetal rat. On day 20, female pups had rates of TEWL that were $40 \%$ lower than those of males. Oestrogens accelerated and testosterone retarded epidermal barrier maturation both structurally and functionally, when studied in vitro or in vivo. Similar effects of oestrogen and testosterone on

\section{Key messages}

- Epidermal maturation is strongly dependent on gestation. High transepidermal water loss (TEWL) is the major consequence of an immature barrier

- Antenatal steroids and sex have a strong maturational effect on the epidermis in the experimental animal

- In the human preterm infant, no maturational effect of antenatal steroids or sex can be shown

- Alternative strategies are needed to manage the high TEWL of the most immature infants

pulmonary maturation have been noted. ${ }^{22-24}$ The antiandrogen flutamide abolished sex differences in epidermal barrier maturation, just as it has been shown to abolish sex differences in pulmonary maturation. ${ }^{24}$ We have failed to show any sex difference in epidermal barrier maturation in the human preterm infant although the influence of sex on pulmonary maturation is well known. ${ }^{25}{ }^{26}$ The latter is relatively small in comparison with the influence of antenatal steroids in the human. Although our study did not have the power to detect a small sex effect on TEWL, again it seems likely that a species difference explains our failure to show an effect.

The clinical problems associated with epidermal barrier immaturity remain. As postnatal existence results in rapid maturation, these problems are transient. Attention needs to be focused on alternative ways of overcoming them such as environmental manipulation or the use of emollients in the immediate newborn period.

1 Holbrook KA. Human epidermal embryogenesis. Int $f$ Dermatol 1979;18:329-56.

2 Evans NJ, Rutter N. Development of the epidermis in the Evans NJ, Rutter N. Development of the
newborn. Biol Neonate 1986;49:74-80.

3 Hammarlund K, Sedin G. Transepidermal water loss in newborn infants. III. Relation to gestational age. Acta Paediatr 1979;68:795-801.

4 Rutter N, Hull D. Water loss from the skin of term and preterm babies. Arch Dis Child 1979;54:858-68.

5 Rutter N. Percutaneous drug absorption in the newborn: hazards and uses. Clin Perinatol 1987;14:911-30.

6 Elias PM, Menon GK. Structural and lipid biochemical correlates of the epidermal permeability barrier. Adv Lipid Res 1991;24:1-26.

7 Aszterbaum M, Feingold KR, Menon GK, Williams ML. Glucocorticoids accelerate fetal maturation of the epidermal permeability barrier in the rat. $f$ Clin Invest 1993;91:2703-8.

8 Okah FA, Pickens WL, Hoath SB. Effect of prenatal steroids on skin surface hydrophobicity in the premature rat. Pedion skin surface hydroph

9 Hanley K, Rassner U, Jiang Y, et al. Hormonal basis for the gender difference in epidermal barrier formation in the gender difference in epidermal barrier formation in the
fetal rat. Acceleration by estrogen and delay by testosterfetal rat. Acceleration by estrogen an
one. $\mathcal{F}$ Clin Invest 1996;97:2576-84.

10 Nilsson GE. Measurement of water exchange through skin. Med Biol Eng Comput 1977;15:209-18.

11 Hammarlund K, Nilsson GE, Oberg PA, Sedin G. Transepidermal water loss in newborn infants. I. Relation to ambient humidity and site of measurement and estimation of total transepidermal water loss. Acta Paediatr 1977;66:553-62.

12 Hammarlund K, Sedin G, Stromberg B. Transepidermal water loss in newborn infants. VII. Relation to post-natal age in very pre-term and full-term appropriate for gestational age infants. Acta Paediatr 1982;71:369-74

13 Cartlidge PHT, Rutter N. Skin barrier function. In: Polin RA, Fox WW, eds. Fetal and neonatal physiology, 2nd ed. RA, Fox WW, eds. Fetal and neonatal physioh

14 Pang KY, Newman AP, Udall JN, Walker WA. Development of gastrointestinal mucosal barrier. VII. In utero maturation of microvillus surface by cortisone. Am f Physiol tion of microvillus 
15 Trahair JF, Perry RA, Silver M, Robinson PM. Studies on the maturation of the small intestine in the fetal sheep. II. The

16 Baum M, Quigley R. Prenatal glucocorticoids stimulate neonatal juxtamedullary proximal convoluted tubule acidification. Am F Physiol 1991;261:F746-52

17 Celsi G, Wang ZM, Akusjarvi G, Aperia A. Sensitive periods for glucocorticoids' regulation of $\mathrm{Na}^{+}, \mathrm{K}^{+}$-ATPase mRNA in the developing lung and kidney. Pediatr Res 1993;33:5-9.

18 Dodic M, Wintour EM. Effects of prolonged $(48 \mathrm{~h})$ infusion of cortisol on blood pressure, renal function and fetal fluids in the immature ovine foetus. Clin Exp Pharmacol Physiol 1994;21:971-80.

19 Aszterbaum M, Menon GK, Feingold KR, Williams ML. Ontogeny of the epidermal barrier to water loss in the rat: correlation of function with stratum corneum structure and lipid content. Pediatr Res 1992;31:308-17.

20 Agren J, Siors G, Sedin G. Transepidermal water loss in infants born at 24 and 25 weeks of gestation. Acta Paediatr 1998:87:1185-90.
21 Crowley P, Chalmers I, Keirse MJ. The effects of corticosteroid administration before preterm delivery: an corticosteroid administration before preterm delivery: an
overview of the evidence from controlled trials. $\mathrm{BrF}$ Obstet Gynaecol 1990;97:11-25.

22 Khosla SS, Smith GJ, Parks PA, Rooney SA. Effects of estrogen on fetal rabbit lung maturation: morphological and biochemical studies. Pediatr Res 1981;15:1274-81.

23 Nielsen HC, Zinman HM, Torday JS. Dihydrotestosterone inhibits fetal rabbit pulmonary surfactant production. $f$ Clin Invest 1982;69:611-16.

24 Torday JS. Androgens delay human fetal lung maturation in vitro. Endocrinology 1990;126:3240-4.

25 Khoury MJ, Marks JS, McCarthy BJ, Zaro SM. Factors affecting the sex differential in neonatal mortality: the role of respiratory distress syndrome. Am 7 Obstet Gynecol 1985;151:777-82.

26 La Pine TR, Jackson JC, Bennett FC. Outcome of infants weighing less than 800 grams at birth: 15 years' weighing less than 800 grams at
experience. Pediatrics 1995;96:479-83. 